\title{
Absolute Internal Energy of the Real Gas
}

\author{
Albrecht Elsner \\ Am Mühlbach, Garching, Germany \\ Email: alimeli.elsner@gmail.com
}

How to cite this paper: Elsner, A. (2017) Absolute Internal Energy of the Real Gas. Engineering, 9, 361-375.

https://doi.org/10.4236/eng.2017.94020

Received: March 22, 2017

Accepted: April 27, 2017

Published: April 30, 2017

Copyright (C 2017 by author and Scientific Research Publishing Inc. This work is licensed under the Creative Commons Attribution International License (CC BY 4.0).

http://creativecommons.org/licenses/by/4.0/

\begin{abstract}
The internal energy $U$ of the real, neutral-gas particles of total mass $M$ in the volume $V$ can have positive and negative values, whose regions are identified in the state chart of the gas. Depending on the relations among gas temperature $T$, pressure $p$ and mass-specific volume $v=V / M$, the mass exists as a uniform gas of freely-moving particles having positive values $U$ or as more or less structured matter with negative values $U$. In the regions $U>0$ above the critical point $\left[T_{c}, p_{c}, \nu_{c}\right]$ it holds that $p(T, v)>p_{c}$ and $v>v_{c}$, and below the critical point it holds that $p(T, v)<p_{c}$ and $v>v_{v}$,
\end{abstract} where $v_{v}$ is the mass-specific volume of saturated vapor. In the adjacent regions with negative internal energy values $U<0$ the mean distances between particles are short enough to yield negative energy contributions to $U$ due to inter-particle attraction that exceeds the thermal, positive energy contributions due to particle motion. The critical isochor $v_{c}$ is the line of equal positive and negative energy contributions and thus represents a line of vanishing internal energy $U=0$. At this level along the critical isochor the ever present microscopic fluctuations in energy and density become macroscopic fluctuations as the pressure decreases on approaching the critical point; these are to be observed in experiments on the critical opalescence. Crossing the isochor $v_{c}$ from $U>0$ to $U<0$, the change in energy $\Delta U>0$ happens without any discontinuity. The saturation line $v_{v}$ also separates the regions between $U>0$ and $U<0$, but does not represent a line $U=0$. The internal-energy values of saturated vapor $U_{v}$ and condensate $U_{1}$ can be determined absolutely as functions of vapor pressure $p$ and densities $(M / V)_{v}$ and $(M / V)_{l}$, repectively, yielding the results $U_{l}<0<U_{v}, U=U_{l}+U_{v}<0$ for $T<T_{c}$ and $U=U_{l}=U_{v}=0$ at the critical point. Crossing the line $v_{v}$ from $U=U_{v}>0$ to $U=U_{v}+U_{l}<0$ requires the energy $\Delta U=-U_{l}>0$ to be removed from the particle system. The ther- modynamic and quantum-mechanical formulations of the internal energy of a particle system only agree if both the macroscopic and microscopic energy scales have the same absolute energy reference value 0 . 
Arguments for the energy reference value $U=0$ in the state of transition from bound to freely- moving particles in macroscopic classical and microscopic quantum particle systems are discussed.

\section{Keywords}

Energy Reference Value Zero in Microscopic and Macroscopic Particle Systems, State of Transition from Bound to Freely-Moving Particles, Internal Energy Regions in the State Chart of Gas, Critical Point and Critical Isochor Loci of Vanishing Internal Energy, Critical Opalescence, BEC, Calculation of Internal Energies of Saturated Liquid and Vapor

\section{Introduction}

Investigated here are thermodynamic properties of a real single-component gas of mass $M$ in the volume $V$. In accordance with Gibbs [1], the internal energy $U$ is assigned to the gas, composed of a very large number of particles, and the entropy $S$ to its distribution in $V$. It is not the total energy of $M$ in $V$ that is considered, i.e. not the contributions from relativistic or kinetic and potential energies of a mass system moving in external fields, but only the contributions resulting from all time-independent kinetic and potential energies in $V$ averaged over all particles. The sum of energies exchanged between the particles can take a positive, negative or else vanishing value $U$, depending on the conditions to which the gas is subjected.

\section{Dependence of the Sign of the Internal Energy of the Real Gas on the Aggregate State of Its Mass}

Phenomenologically, a distinction is to be made between three thermodynamic conditions under which the gas is in equilibrium. Thermodynamic equilibrium states that the fluid particles are homogeneously distributed in the particular phase volumes and satisfy there the Boltzmann equation, i.e. the equation of kinetic theory. By means of variation of the external parameters, temperature and pressure, the mean distance $d$ between fluid particles, which are continually in motion, can be varied; this distance determines the statistical mean value of both the thermal energy and the attractive energy of the particles in each phase volume and is thus related to the aggregate state of the particular fluid mass. In the following, the ratio of the mean distance between fluid particles at given temperature, pressure and volume to that assumed at the critical state, is denoted as $r=d_{T, p, v} / d_{T_{c}, p_{c}, v_{c}}=\left(v / v_{c}\right)^{1 / 3}$.

1) Above the critical point for pressures $p \geq p_{c}$ and volumes $V / M=v>v_{c}$ and below the critical point for pressures $p<p_{c}$ and volumes $v>v_{v}$, where $v_{v}$ is the mass-specific volume of vapor, the mass $M$ exists as a gas homogeneously distributed in $V$. For values $r>1$, the averaged kinetic energies of all freely moving particles exceed their mutual bindings, which as potential attractive energy contributions are to be regarded as negative, so that 


$$
U(M)>0 \quad \text { for gaseous mass }(r>1) .
$$

2) Starting from the condition at the critical point $\left[T_{c}, p_{c}, v_{c}\right]$, at temperatures $T<T_{c}$ and pressures $p<p_{c}$, the mass $M$ disintegrates into a vapor mass $M_{v}$ with particle volume $\left(V_{v} / M_{v}\right)=v_{v}>v_{c}$ and a condensed mass $M_{l}$ with $\left(V_{l} / M_{l}\right)=v_{l}<v_{c}$. Under the equilibrium condition $r<1$ one has the relations $V=V_{v}+V_{l}$ and $M=M_{v}+M_{l}$, where $M_{v}<M_{l}=M-M_{v}$ or $M_{v}<M / 2<M_{l}$. The internal energy of all freely moving vapor particles is positive, i.e. $U\left(M_{v}\right)>0$, and that of the more closely bound, not freely moving, condensed particles is negative, i.e. $U\left(M_{l}\right)<0$. The internal energy of the gas mass $M$ is likewise negative owing to the excess of more closely bound condensed particles. From $\left|U\left(M_{l}\right)\right| / U\left(M_{v}\right)>1$ one obtains $U(M)=U\left(M_{v}\right)+U\left(M_{l}\right)<0$. It then follows that

$$
U(M)<0 \quad \text { for vapor mass }(r>1) \text { and condensate mass }(r<1) .
$$

3) At the critical point the masses $M_{v}$ and $M_{l}$ are equally large, i.e. $M_{v}=M / 2=M_{l}$. The internal energies are likewise equally large, i.e. $U\left(M_{v}\right)=U(M / 2)=U\left(M_{l}\right)$. Under the condition $r=1$ the energy contributions of motion and binding cancel out in sum, making it impossible to distinguish between positive and negative values, so that $U\left(M_{v}\right)=U\left(M_{l}\right)=0$. At the critical point, however, not only do the values of the internal energies of vapor and condensate vanish but, since $U(M)=U\left(M_{v}\right)+U\left(M_{l}\right)$, the energy value of the total fluid mass does as well. The result is

$$
U(M)=0 \quad \text { for mass under critical conditions }(r=1) \text {. }
$$

The sign of the internal energy $U$ of the real gas depends on the aggregate state of its mass $M$. The aggregate state, on the other hand, is determined by the mean distances $d$ between fluid particles, which are qualitatively described by the value of the relative number $r=d_{T, p, v} / d_{T_{c}, p_{c}, v_{c}}$.

\section{Macroscopic Energy and Density Fluctuations in the Fluid on Approaching the Critical Point}

In a fluid, macroscopic density fluctuations are observed in a narrow temperature range before the critical temperature is reached [2]. Andrews discovered these for the first time in 1869 when he irradiated a gas $\left(\mathrm{CO}_{2}\right)$ with visible light (wavelengths $\lambda=4 \times 10^{-7} \sim 7 \times 10^{-7}[\mathrm{~m}]$ ) and found that the previously transparent gas became opaque (within $\left.\left(T-T_{c}\right) \approx 10^{-3} T_{c}\right)$; this phenomenon has since been known as critical opalescence. Macroscopic fluctuations of mass indicate instability of the gas as the consequence of the vanishing internal energy $U=0$ on approaching the critical point.

In the following, the physical processes occuring when the gas approaches the critical point are investigated. In the course of cooling the kinetic energy of all gas particles decreases and at the same time their binding energy increases. This also raises the probability that particles with diameters of the order $10^{-10}[\mathrm{~m}]$ aggregate to more voluminous mass conglomerations with diameters of light wavelengths. From their surfaces the light is reflected and scattered proportionally 
to $\lambda^{4}$ in different directions. The deflection intensity increases in the course of cooling with the number and size of the scattering centres [2]. The illuminated fluid appears opaque and does not become transparent again till the critical point is reached because there a single aggregate with about $10^{-2}[\mathrm{~m}]$ diameter and vapor particles with diameters of less than $10^{-9}[\mathrm{~m}]$ exist from which visible light is no longer diffusely scattered.

The reason why there are mass fluctuations in the scattering range between $10^{-6}[\mathrm{~m}]$ and $10^{-4}[\mathrm{~m}][2]$ is associated with the macroscopic fluctuations of the internal energy of the real gas at the critical point. The deviation of the fluctuating quantity is considered, $\tilde{U}-U$; its mean value vanishes owing to equally large positive and negative deviations from $U$, i.e. $\langle\tilde{U}-U\rangle=0$. As a convenient measure of the amount of scatter of the value $U$ is the mean value of the always positive quadratic deviation $\left\langle(\tilde{U}-U)^{2}\right\rangle$. Because one has $U=0$, the mean value of the square of the energy fluctuations occurring is [3]

$$
\left\langle(\tilde{U}-U)^{2}\right\rangle=\left\langle(\tilde{U})^{2}\right\rangle>0
$$

The square root $\sqrt{\left\langle(\tilde{U})^{2}\right\rangle}$ gives the standard deviation of $U$ near the critical point. The fluctuations of energy are thermodynamically associated with fluctuations of mass. These spread out in the fluid on approaching the critical point, becoming stronger and slower because under the condition of decreasing pressure in the fluid there is an increasing volume for fluctuations disposable.

In a Bose gas the phenomenon of critical opalescence is also observed. The shining of the gas cloud illuminated by laser light appears immediately before its condensation at the critical temperature.

Cooling of a Bose gas from room temperature to values of the critical temperature of $10^{-6}[\mathrm{~K}]$ within minutes occurs through interaction between laser light and gas atoms. By virtue of the fact that the ensuing aggregating high-energy particles are immediately removed from the available volume $V$ there is no coexistent liquid phase. As soon as the low-energy gas particles remaining in $V$ have on average the separation at which attractive and repulsive interatomic forces balance one another, the state of vanishing internal energy sets in. The enhanced macroscopic energy and particle density fluctuations show critical opalescence in the laser light. On the assumption that the fluctuations occur above the critical value $T_{\mathrm{BEC}}$ at a temperature difference of $\left(T-T_{\mathrm{BEC}}\right) \approx 10^{-3} \cdot T_{\mathrm{BEC}}$ there is spontaneous condensation of the total gas mass at a temperature of just $10^{-9}[\mathrm{~K}]$ before the transition temperature $T_{\mathrm{BEC}}$ is reached.

The values of $M, V, U$ and $S$ of both the gas and condensate are needed to describe the real Bose-Einstein gas. These values are thermodynamically defined by the experimental conditions and are given by Equation (7.3) in Section 7.

In 1925 condensation was previously predicted by Einstein for Bose particles: in the lowest quantum mechanical state these assume vanishing entropy and take up equal, lowest possible energy. The first macroscopic quantum phenomenon, 
which was interpreted in 1938 by $\mathrm{H}$. London as Bose-Einstein-condensate, is suprafluid helium. In 1995 Wieman, Cornell and Ketterle succeeded in the first experimental realisations of heavier alkali atoms. The laboratory conditions for a Bose-Einstein-condensate (BEC) call for temperatures in the range of $10^{-6}[\mathrm{~K}]$ and particle densities of $10^{20}$ [part $\left./ \mathrm{m}^{3}\right]$. The particles are isolated from vessel walls in a magneto-optical trap (diameter $10^{-2}[\mathrm{~m}]$ ) and then cooled by means of laser light $\left(\lambda \approx 5 \times 10^{-7}[\mathrm{~m}]\right)$ to get BEC [4].

\section{Thermodynamic Verification of the Value $U=0$ for the Internal Energy at the Critical Point}

In accordance with the thermodynamic theory of Willard Gibbs [1] it is possible to calculate every thermodynamic property of a real gas. The quantities uniquely determining the gas are the absolute values of mass $M$, volume $V$, internal energy $U$ and entropy $S$, which represent the external or extensive parameters of the gas system. The likewise absolute quantities, temperature $T$, pressure $p$ and chemical potential $\mu$, are referred to as intensive parameters. They are defined by $T=(\partial U / \partial S)_{M, V}, \quad p=-(\partial U / \partial V)_{S, M}, \quad \mu=(\partial U / \partial M)_{V, S}$. By measuring the three function values $[T, p, \mu]$ one knows the equilibrium state of the gas in $V$, which is also described by Gibbs's fundamental equation [3]

$$
U+V p-S T-M \mu=0 .
$$

Although Gibbs's theory [1] was already known in the scientific world at the end of the 19th century and the question of absolute values of $U$ and $S$ had been posed, apparently few scientists were interested in tackling it. It was Nernst, Planck, Boltzmann and physicists in quantum mechanics who solved the problem of the thermodynamic reference point for $S$. The corresponding problem for $U$, on the other hand, remained unsolved [2] [5]. Instead, it sufficed to present determinations of arbitrary zero points, e.g. with $S_{l} / M_{l}\left(T_{t}\right)=0$ and $U_{l} / M_{l}\left(T_{t}\right)=0$ at the triple point of a liquid without ever taking thermodynamic conditions into account. Consequently, inconsistencies cropped up; in particular, the free energy $U-S T$ and the chemical potential $U-S T+V p$ were indeterminable functions owing to the missing thermodynamic reference points, so that the fundamental equation could not be applied to every thermodynamic question. Meanwhile the situation has been changed by the proof of $U=0$ found at the critical point [6].

The theory provides the Gibbs-Duhem equations as the sum of the products of the extensive parameters and the differentials of the corresponding intensive parameters [3]. By enlisting the fundamental equation one then obtains the explicit expressions for the internal energy and entropy [3]:

$$
U=\left.M \frac{\partial(\mu / T)}{\partial(1 / T)}\right|_{M, V}-\left.V \frac{\partial(p / T)}{\partial(1 / T)}\right|_{M, V} \quad \text { and } \quad S=-\left.M \frac{\partial \mu}{\partial T}\right|_{M, V}+\left.V \frac{\partial p}{\partial T}\right|_{M, V}
$$

For the saturated fluid, whose two phases vapor and condensate have the same values $p=p(T)$ and $\mu=\mu(T)$ everywhere in $V$, it is immediately possible to give the temperature dependence of the heat capacity: 


$$
C(M, V, T)=\mathrm{d} U / \mathrm{d} T=\mathrm{d} S / \mathrm{d} T T=-M\left(\mathrm{~d}^{2} \mu / \mathrm{d} T^{2}\right) T+V\left(\mathrm{~d}^{2} p / \mathrm{d} T^{2}\right) T .
$$

This relation states that in the case of the saturated fluid there is no difference between a heat capacity at constant volume and one at constant pressure.

In the following, it is to be proved once again that the zero value of $U$ is thermodynamically fixed by Gibbs's theory as $U=0$ at the critical point.

We consider a saturated fluid which according to Gibbs's phase rule has one thermodynamic degree of freedom fewer than the single phase gas and is thus theoretically simpler to treat. The saturated fluid with the two phases, vapor and condensate, then has the critical density $(M / V)$ and the mass-specific critical volume $v_{c}=(V / M)_{c}$. As the fluid values $[T, p, \mu]$ in $V$ are locally equal in each of the particular volumes, the two phases can only be distinguished by the densities or mass-specific volumes $v_{v}=V_{v} / M_{v}$ and $v_{l}=V_{l} / M_{l}$. In the temperature range below the critical point $\left[T_{c}, p_{c}, v_{c}\right]$ one has the relations

$$
\begin{gathered}
M=M_{v}(T)+M_{l}(T), \quad V=V_{v}(T)+V_{l}(T), \\
v_{v}\left(T^{\prime}\right)>v_{v}(T)>v_{v}\left(T_{c}\right)=v_{c}=v_{l}\left(T_{c}\right)>v_{l}(T)>v_{l}\left(T^{\prime}\right)>0 \text { for } T^{\prime}<T<T_{c} .
\end{gathered}
$$

The relative mass distributions with the value $\frac{1}{2}$ at the critical point are

$$
0 \leq \frac{M_{v}(T)}{M}=\frac{v_{c}-v_{l}(T)}{v_{v}(T)-v_{l}(T)} \leq \frac{1}{2} \leq \frac{v_{v}(T)-v_{c}}{v_{v}(T)-v_{l}(T)}=\frac{M_{l}(T)}{M} \leq 1 .
$$

In accordance with the masses the values of the internal energy and entropy in the partial volumens $V_{v}$ and $V_{l}$ are divided as follows:

$$
U(M, V, T)=U_{v}(T)+U_{l}(T), \quad S(M, V, T)=S_{v}(T)+S_{l}(T),
$$

where we abbreviate by putting, for example, $U_{v}(T)$ instead of $U_{v}\left(M_{v}, V_{v}, T\right)$. The mass-specific quantities are

$u(v, T)=U(M, V, T) / M, \quad u_{v}(T)=U_{v}(T) / M_{v}(T), \quad u_{l}(T)=U_{l}(T) / M_{l}(T)$, $s(v, T)=S(M, V, T) / M, \quad s_{v}(T)=S_{v}(T) / M_{v}(T), \quad s_{l}(T)=S_{l}(T) / M_{l}(T)$.

Whereas $u$ is a function of the variables $v$ and $T$, the quantities $u_{v}$ and $u_{l}$ are pure temperature functions since, for example, it holds that $U_{v}\left(M_{v}, V_{v}, T\right) / M_{v}=u_{v}\left(1, V_{v} / M_{v}, T\right)=u_{v}\left(1, v_{v}(T), T\right)=u_{v}(T)$.

The quantity $u\left(v_{c}, T\right)$ can be represented by the quantities $u_{v}(T)$ and $u_{l}(T)$ as follows:

$$
u\left(v_{c}, T\right)=\frac{\mathrm{d}(\mu / T)}{\mathrm{d}(1 / T)}-v_{c} \frac{\mathrm{d}(p / T)}{\mathrm{d}(1 / T)}=\frac{M_{v}}{M} u_{v}+\frac{M_{l}}{M} u_{l}=\frac{\left(v_{c}-v_{l}\right) u_{v}+\left(v_{v}-v_{c}\right) u_{l}}{v_{v}-v_{l}}
$$

This representation is possible if, by analogy with Equation (4.2) for $U$, also the functions $U_{v}=M_{v} u_{v}$ and $U_{l}=M_{l} u_{l}$ are expressed as

$$
\begin{aligned}
& U=M \frac{\mathrm{d}(\mu / T)}{\mathrm{d}(1 / T)}-V \frac{\mathrm{d}(p / T)}{\mathrm{d}(1 / T)}, \\
& U_{v}=M_{v} \frac{\mathrm{d}(\mu / T)}{\mathrm{d}(1 / T)}-V_{v} \frac{\mathrm{d}(p / T)}{\mathrm{d}(1 / T)}, \\
& U_{l}=M_{l} \frac{\mathrm{d}(\mu / T)}{\mathrm{d}(1 / T)}-V_{l} \frac{\mathrm{d}(p / T)}{\mathrm{d}(1 / T)} .
\end{aligned}
$$


The system of Equation (4.10) provides three relations for determining the reference value for the internal energy. Discussion of this is conducted through the mass-specific quantities $u, u_{v}$ and $u_{l}$, whose temperature dependences are described as follows:

$$
\begin{aligned}
& u\left(v_{c}, T\right)=\frac{\mathrm{d}(\mu / T)}{\mathrm{d}(1 / T)}-v_{c} \frac{\mathrm{d}(p / T)}{\mathrm{d}(1 / T)}, \\
& u_{v}(T)=\frac{\mathrm{d}(\mu / T)}{\mathrm{d}(1 / T)}-v_{v}(T) \frac{\mathrm{d}(p / T)}{\mathrm{d}(1 / T)}, \\
& u_{l}(T)=\frac{\mathrm{d}(\mu / T)}{\mathrm{d}(1 / T)}-v_{l}(T) \frac{\mathrm{d}(p / T)}{\mathrm{d}(1 / T)}
\end{aligned}
$$

The difference $\left(u_{v}-u_{l}\right)$ yields the Clapeyron-Clausius equation:

$$
u_{v}-u_{l}=-\left(v_{v}-v_{l}\right) \mathrm{d}(p / T) / \mathrm{d}(1 / T) \text {. }
$$

From $v_{v}-v_{l} \geq 0$ and $\mathrm{d}(p / T) / \mathrm{d}(1 / T)=p-\mathrm{d} p / \mathrm{d} T T<0$ it follows that $u_{v}-u_{l} \geq 0$ and $u_{v} \geq u_{l}$. At the critical point one has $u_{v}=u_{l}$, i.e. the value of the evaporation energy $\left(u_{v}-u_{l}\right)(T)$ is always positive and vanishes at $T_{c}$. As $u=u_{v}+\left(v_{v}-v_{c}\right) \mathrm{d}(p / T) / \mathrm{d}(1 / T)=u_{l}-\left(v_{c}-v_{l}\right) \mathrm{d}(p / T) / \mathrm{d}(1 / T)$, the value of $u\left(v_{c}, T\right)$ is smaller than that of $u_{v}$ and larger than $u_{l}$ and at $T_{c}$ takes the value $u_{v}=u_{l}$, which is denoted here as $u_{c}$. This gives the relations

$$
u_{l}(T) \leq u\left(v_{c}, T\right) \leq u_{c} \leq u_{v}(T) \text { for } 0 \leq T \leq T_{c} .
$$

It is now to be shown that $u_{c}=0$ is valid. Because the stated extensive and intensive parameters are absolute quantities, $u_{c}$ has an absolute value. It is certainly reasonable to assume this to be zero for the time being since otherwise choosing a positive or negative value should be physically justified so as not to appear arbitrary. With the choice $u_{c}=0$ it follows from the three Equation (4.11) that

$$
\left.\frac{\mathrm{d}(\mu / T)}{\mathrm{d}(1 / T)}\right|_{c}=\left.v_{c} \frac{\mathrm{d}(p / T)}{\mathrm{d}(1 / T)}\right|_{c}<0 \text { for } T=T_{c}
$$

This relation can be enlisted as definition of the critical volume,

$$
\begin{gathered}
\mathrm{d}(\mu / T) /\left.\mathrm{d}(1 / T)\right|_{c} / \mathrm{d}(p / T) /\left.\mathrm{d}(1 / T)\right|_{c}=v_{c}>0 \text {. Relations }(4.13) \text { then read } \\
u_{l}=\mathrm{d}(\mu / T) / \mathrm{d}(1 / T)-v_{l} \mathrm{~d}(p / T) / \mathrm{d}(1 / T) \leq u \\
u=\mathrm{d}(\mu / T) / \mathrm{d}(1 / T)-\mathrm{d}(\mu / T) /\left.\mathrm{d}(1 / T)\right|_{c} / \mathrm{d}(p / T) /\left.\mathrm{d}(1 / T)\right|_{c} \mathrm{~d}(p / T) / \mathrm{d}(1 / T) \leq 0 \\
0 \leq \mathrm{d}(\mu / T) / \mathrm{d}(1 / T)-v_{v} \mathrm{~d}(p / T) / \mathrm{d}(1 / T)=u_{v} .
\end{gathered}
$$

If the volume function

$$
\bar{v}=\frac{\mathrm{d}(\mu / T) / \mathrm{d}(1 / T)}{\mathrm{d}(p / T) / \mathrm{d}(1 / T)}=\frac{\mathrm{d}(\mu / T)}{\mathrm{d}(p / T)}>0 \quad \text { for } 0 \leq T \leq T_{c}
$$

is introduced, one can give as upper and lower limits for $\bar{v}$ the temperature functions $v_{v}(T)$ and $\frac{1}{2}\left(v_{v}(T)+v_{l}(T)\right)$, which at $T_{c}$ have the value $v_{c}$ and with decreasing temperature increase monotonically. This shows that the differential quotient of the chemical potential $d(\mu / T) / d(1 / T)$ is a negative 
temperature function which with increasing values $T$ monotonically increases to the negative value $v_{c} \mathrm{~d}(p / T) /\left.\mathrm{d}(1 / T)\right|_{c}$ at $T_{c}$.

The mass-specific energies can thus be put in the simpler form:

$$
\begin{gathered}
u_{l}=\left[\bar{v}-v_{l}\right] \mathrm{d}(p / T) / \mathrm{d}(1 / T) \leq u=\left[\bar{v}-v_{c}\right] \mathrm{d}(p / T) / \mathrm{d}(1 / T) \leq 0, \\
0 \leq\left[\bar{v}-v_{v}\right] \mathrm{d}(p / T) / \mathrm{d}(1 / T)=u_{v} .
\end{gathered}
$$

It can be shown that the volume function $\bar{v}$ can be explicitly represented by the following thermodynamic expression [7]:

$$
\bar{v}=\bar{v}\left(v_{v}, v_{l}\right)=v_{v}+v_{l}-\left(v_{v}-v_{l}\right) / \ln \left(v_{v} / v_{l}\right)=\bar{v}\left(v_{l}, v_{v}\right) \geq v_{c} .
$$

The symmetric occurrence of the variables $v_{v}$ and $v_{l}$ in $\bar{v}$ marks the phase independence of the volume function $\mathrm{d}(\mu / T) / \mathrm{d}(p / T)$. The internal energies can now be expressed in terms of the measurable quantities $p$ and $v_{v, l}:$

$$
\begin{gathered}
u_{l}=\left[v_{v}-\left(v_{v}-v_{l}\right) / \ln \left(v_{v} / v_{l}\right)\right] \mathrm{d}(p / T) / \mathrm{d}(1 / T) \leq u, \\
u=\left[v_{v}+v_{l}-\left(v_{v}-v_{l}\right) / \ln \left(v_{v} / v_{l}\right)-v_{c}\right] \mathrm{d}(p / T) / \mathrm{d}(1 / T) \leq 0, \\
0 \leq u_{v}=\left[v_{l}-\left(v_{v}-v_{l}\right) / \ln \left(v_{v} / v_{l}\right)\right] \mathrm{d}(p / T) / \mathrm{d}(1 / T) .
\end{gathered}
$$

Energies as extensive parameters can be added and subtracted. From

$2 u_{v}-\left(u_{v}-u_{l}\right)=2 u_{l}+\left(u_{v}-u_{l}\right)=\left(u_{v}+u_{l}\right)=\left[2\left(v_{v}-v_{l}\right) / \ln \left(v_{v} / v_{l}\right)-v_{v}-v_{l}\right]$ $(-\mathrm{d}(p / T) / \mathrm{d}(1 / T))=u+\left[\left(v_{v}-v_{l}\right) / \ln \left(v_{v} / v_{l}\right)-v_{c}\right](-\mathrm{d}(p / T) / \mathrm{d}(1 / T)) \leq 0$ follows in addition to relations (4.13) and (4.19) that

$$
-\frac{1}{2}\left(u_{v}-u_{l}\right) \leq u_{l} \leq u \leq\left(u_{v}+u_{l}\right) \leq 0 \leq u_{v} \leq \frac{1}{2}\left(u_{v}-u_{l}\right) .
$$

Particularly interesting is the ratio of $u_{v}$ to $u_{l}$, which as a function of the volume ratio $z=v_{v} / v_{l}$ assumes universally for every gas values between -1 at the critical point and 0 at absolute zero:

$$
-1 \leq \frac{u_{v}}{u_{l}}=\frac{v_{l}-\left(v_{v}-v_{l}\right) / \ln \left(v_{v} / v_{l}\right)}{v_{v}-\left(v_{v}-v_{l}\right) / \ln \left(v_{v} / v_{l}\right)}=\frac{z-1-\ln z}{z-1-z \ln z} \leq 0 \quad \text { for } z=\frac{v_{v}}{v_{l}} \geq 1 \text {. }
$$

From this it follows that the ratio of the phase-specific energies to the evaporation energy is likewise universal:

$$
0 \leq \frac{u_{v}}{u_{v}-u_{l}}=\frac{z-1-\ln z}{(z-1) \ln z} \leq \frac{1}{2} \leq \frac{z \ln z-(z-1)}{(z-1) \ln z}=\frac{\left(-u_{l}\right)}{u_{v}-u_{l}} \leq 1 .
$$

It can readily be shown that the internal energy of the saturated fluid $U=U_{l}+U_{v}$ is not positive. Equations (4.4), (4.6), (4.12) and (4.19) yield $U=\left(M-M_{v}\right) u_{l}+M_{v} u_{v}=M u_{l}+M_{v}\left(u_{v}-u_{l}\right)$

$$
=M\left[v_{v}-\left(v_{v}-v_{l}\right) / \ln \left(v_{v} / v_{l}\right)-\left(v_{c}-v_{l}\right)\right] \mathrm{d}(p / T) / \mathrm{d}(1 / T)=M u \leq 0 \text {. From this }
$$

one obtains

$$
\begin{gathered}
u_{v}+u_{l}=\left[v_{v}+v_{l}-2\left(v_{v}-v_{l}\right) / \ln \left(v_{v} / v_{l}\right)\right] \mathrm{d}(p / T) / \mathrm{d}(1 / T) \leq 0, \\
U_{v}+U_{l}=M\left[v_{v}+v_{l}-\left(v_{v}-v_{l}\right) / \ln \left(v_{v} / v_{l}\right)-v_{c}\right] \mathrm{d}(p / T) / \mathrm{d}(1 / T)=M u \leq 0, \\
-1 \leq \frac{u_{v}}{u_{l}} \leq \frac{U_{v}}{U_{l}}=\frac{u_{v}}{u_{l}} \frac{M_{v}}{M_{l}} \leq 0 \quad \text { for } T_{c} \geq T \geq 0 \text { and } 1 \geq \frac{M_{v}}{M_{l}} \geq 0 .
\end{gathered}
$$


The above mentioned relations (4.14)-(4.23) are a consequence of fixing the critical value $u_{c}=0$. If, on the other hand, the assumption $u_{c}=\Delta \neq 0$ were correct, relations (4.13) would read $u_{l}+\Delta \leq u+\Delta \leq \Delta \leq u_{v}+\Delta$ and a unique transformation between thermodynamic parameters would no longer be given; one need only think of the functions, free energy and chemical potential, which would then remain undetermined because of the constant $\Delta \neq 0$, whereas it is just these functions, which regulate all processes in nature, that should be determinable.

$\Delta \neq 0$ is not compatible with the extensivity of $U$ formulated by Gibbs, but it holds that $U\left(M, V, T_{c}\right)=0$. This then also means that the absolute temperature value $U(M, V, T)$ can be measured. If a cooling process starts at $T_{c}$ and ends at $T<T_{c}$ and the energy decrease is measured in the form of the heat removed from the system by the amount $|\Delta Q|, U(M, V, T)$ is negative and is determined from

$$
0<|\Delta Q|=U\left(M, V, T_{c}\right)-U(M, V, T)=-U(M, V, T) .
$$

$U(M, V, T)$ can also be calculated from the heat capacity $C(M, V, T)$. Both functions $U(M, V, T)$ and $C(M, V, T)$ have a discontinuity at the triple point and increase monotonically as $T$ :

$$
\begin{aligned}
U(M, V, T) & =-\int_{U}^{U_{c}} \mathrm{~d} U(M, V, T)=-\int_{T}^{T_{c}} \mathrm{~d} U(M, V, T) / \mathrm{d} T d T \\
& =-\int_{T}^{T_{c}} C(M, V, T) \mathrm{d} T \leq 0 \quad \text { for } 0 \leq T \leq T_{c} .
\end{aligned}
$$

The value of the internal energy of the saturated fluid is negative for $T<T_{c}$.

\section{Thermodynamic Verification of the Value $S=0$ for the Entropy at Absolute Zero}

In the context of quantum theory the known Planck form of the Nernst postulate, viz.

$$
S=0 \text { for } T=0,
$$

is interpreted as a macroscopic entropy state of a particle system whose value is proportional to the logarithm of the number of its realized microstates. At the temperature absolute zero the particle system is in the ground state of lowest energy, at which it assumes just a single microstate. In the context of the thermodynamic theory of Willard Gibbs the result (5.1) can likewise be derived.

From $\quad s T=u+v p-\mu \quad$ and $\quad u=\mathrm{d}(\mu / T) / \mathrm{d}(1 / T)-v_{c} \mathrm{~d}(p / T) / \mathrm{d}(1 / T) \quad$ it follows in agreement with Equation (4.2) that

$$
s\left(v_{c}, T\right)=v_{c} \mathrm{~d} p / \mathrm{d} T-\mathrm{d} \mu / \mathrm{d} T,
$$

where $s\left(v_{c}, T\right)$ is an integrable temperature function. Integration between $T=0$ and $T=T_{c}$ with the values $s_{0}$ and $s_{c}$ yields

$$
s_{c}-s_{0}=\int_{s\left(v_{c}, 0\right)}^{s\left(v_{c}, T_{c}\right)} \mathrm{d} s=\int_{0}^{T_{c}} \frac{\mathrm{d}}{\mathrm{d} T}\left[v_{c} \frac{\mathrm{d} p}{\mathrm{~d} T}-\frac{\mathrm{d} \mu}{\mathrm{d} T}\right] \mathrm{d} T=\left.v_{c} \frac{\mathrm{d} p}{\mathrm{~d} T}\right|_{c}-\left.\frac{\mathrm{d} \mu}{\mathrm{d} T}\right|_{c}=s_{c},
$$

because the differential quotients $\mathrm{d} p / \mathrm{d} T$ and $\mathrm{d} \mu / \mathrm{d} T$ vanish at absolute zero, $\lim _{T \rightarrow 0} \mathrm{~d} p / \mathrm{d} T=\mathrm{d} p /\left.\mathrm{d} T\right|_{0}=0$ and $\lim _{T \rightarrow 0} \mathrm{~d} \mu / \mathrm{d} T=\mathrm{d} \mu /\left.\mathrm{d} T\right|_{0}=0$. One therefore 
gets

$$
S(M, V, 0)=V \mathrm{~d} p /\left.\mathrm{d} T\right|_{0}-M \mathrm{~d} \mu /\left.\mathrm{d} T\right|_{0}=0 .
$$

The entropy relation analogous to Equation (4.25) is

$$
\begin{aligned}
S(M, V, T) & =\int_{0}^{S} \mathrm{~d} S(M, V, T)=\int_{0}^{T} \mathrm{~d} S(M, V, T) / \mathrm{d} T \mathrm{~d} T \\
& =\int_{0}^{T} C(M, V, T) / T \mathrm{~d} T \geq 0 \quad \text { for } 0 \leq T \leq T_{c} .
\end{aligned}
$$

\section{Relation between the Internal Potential and Thermal Energies of the Particle System}

By definition, at a given configuration of a particle system the internal energy $U$ of the system is the sum of the binding or potential energy $U_{\text {pot }}$ and the kinetic or thermal energy $U_{\text {th }}$ of all interacting particles, i.e.

$$
U=U_{p o t}+U_{t h} \text {, where } U_{p o t}<0, U_{t h}>0 .
$$

For a certain particle configuration the sum $U_{p o t}+U_{t h}$ thus expresses the law of internal energy conservation. In turn, with the internal energy value given the particle arrangement in the system is fixed and stable. From relations (6.1) it follows that

$$
U_{p o t}=U-U_{t h}<U \text { and } U_{t h}=U-U_{p o t}>U \text {. }
$$

According to relations (2.1)-(2.3) the value of $U$ depends on the mean inter-particle separation $r$ :

$$
U<0 \text { for } r<1, U>0 \text { for } r>1, U=0 \text { for } r=1 .
$$

Relations (6.2) and (6.3) are represented, for example, in Figure 2 of ref. [8] for the two-phase water fluid. In this special case it holds that

$$
U=U_{l}+U_{v} \leq 0 \text {, where } U_{l} \leq 0, U_{v} \geq 0
$$

and

$$
U_{p o t}<U_{l} \leq U=U_{l}+U_{v} \leq 0 \leq U_{v}<U_{t h} .
$$

Relations (6.3) and (6.5) are plotted in the volume-vs.-pressure chart of water in Figure 3 of ref. [8]. The statement reported there that the isotherm $T_{c}$ is a line $U=0$ has to be corrected; instead the isochor $v_{c}$ is the line with $U=0$; this is shown in the following section.

\section{The Conditions $U=0$ and $S=0$}

As the mass $M$ in $V$ can assume both positive and negative values of the internal energy, the question of the course of the line $U=0$ in the state chart arises. In the case $U=0$ the mass $M$ has the entropy value $V(\partial p / \partial T)_{v}-M(\partial \mu / \partial T)_{v}=S=V p / T-M \mu / T$. These equations yield not only the already known relation (4.14) valid at $T=T_{c}$, but they are also valid along the critical isochor $(V / M)_{c}$, where we have the following relation between specific volume $V / M$ and the other intensive parameters, temperature, pressure and chemical potential: 


$$
(\partial(\mu / T) / \partial(p / T))_{v}=V / M=v=v_{c} \quad \text { if } U=0 .
$$

This relation can immediately be derived from Equation (4.2). For $p<p_{c}$ one has either $U<0$ or $U>0$. In the case of the saturated fluid it holds that $U_{l}<0<U_{v}$ and relations (4.16) and (4.18) are valid.

If, on the other hand, the mass $M$ in $V$ has the entropy value $S=0$, then it has the internal-energy value

$M(\partial(\mu / T) / \partial(1 / T))_{v}-V(\partial(p / T) / \partial(1 / T))_{v}=U=M \mu-V p$. This, in turn, yields the Nernst relations (5.4) valid at $T=0$ :

$$
-(\partial \mu / \partial T)_{v}=0,(\partial p / \partial T)_{v}=0,(-\mathrm{d} \mu / \mathrm{d} T / \mathrm{d} p / \mathrm{d} T)_{v}=V / M \quad \text { if } S=0 .
$$

The arrows of $\mu$ in Figure 9.4 and Figure 9.5 of ref. [3] have to be put in the opposite direction to get the correct relation $\mathrm{d} \mu / \mathrm{d} p<0$, since for $T<T_{c}$ the chemical potential $\mu$ is a negative and concavely curved function of pressure $p$ [6].

The thermodynamically established result $U=0$ states that the statistical mean value of the internal energy of a particle system vanishes under the condition of equal energy contributions of binding and repulsion. The same result must be afforded by quantum mechanical calculation of stationary energy states of a particle system because thermodynamics and quantum mechanics are based on the same suppositions when treating a particle system, viz. the conservation of energy and momentum and application of statistical laws [3] [9]. Cohesion of all system particles is accomplished by interatomic interaction of the particles, which are continually in motion. Quantum mechanical calculation of the stationary and stable energy states provides as result the revealing picture of discrete energy steps arranged above one another. The lowest energy step constitutes the ground state of the particle system and the energy levels above specify the particle system completely. A particle system is then in the ground state of lowest energy when its cohesion by binding energy is maximal and the kinetic energy components are minimal. A particle system may consist of a single atom or a multitude of particles. For example, a Bose-Einsteincondensate. From a statistical point of view, bosons are to be treated as undistinguishable particles; consequently, the sum of the lowest particle energy of $N$ condensed bosons, viz. $N u_{\text {boson }}<0$, yields the zero-momentum, ground state energy of the particle system BEC, which is negative and denoted by $U_{\mathrm{BEC}}$. If thermal energy is supplied to a structured particle condensate which can absorb it in discrete energy quanta and step by step can then change the arrangement of its particles, with sufficient excitation energy the energy level is reached at which the binding and excitation energies have equal values and particle cohesion is no longer given. This energy level is assigned the internal energy zero. Above this level system particles move freely and have positive internal energy.

The energy zero of a particle system, $U=0$, is determined by the transition from the bound state particles to freely moving particles. In relation to the number $N$ of a particle system, the negative energy value of the condensate is 
low for a quantum system with $N=1 \rightarrow 100$, which may be quantum mechanically calculated, and high for a macroscopic system with $N$ of order Avogadro numbers, which can only be treated by thermodynamic methods. In other words, the energy reference value $U=0$ is the same for both the microscopic and macroscopic energy scales.

Thermodynamically, the decisive energy transitions in the case BEC can be described as follows: The cold gas mass $M$ of critical temperature $T_{\mathrm{BEC}}$ is contained in the volume $V_{\text {gas }}$ and has the critical density $\rho_{\text {gas }}=M / V_{\text {gas }}$. The presence of critical opalescence indicates the state $U_{\text {gas }}=0$. Under this condition $M$ condenses into the volume $V_{\mathrm{BEC}}<V_{\text {gas }}$. The BEC state is denoted by the density $\rho_{\mathrm{BEC}}=M / V_{\mathrm{BEC}}>\rho_{\text {gas }}$, lowest energy $U_{\mathrm{BEC}}$ and vanishing entropy $S_{\mathrm{BEC}}=0 . T_{\mathrm{BEC}}$ is the lowest, experimentally attainable temperature value. At $T_{\mathrm{BEC}}$ the state of the condensate is more stable than that of the cold gas because in thermodynamic equilibrium the free energy $F_{\mathrm{BEC}}$ is lower than $F_{\text {gas }}$. From $0>F_{\mathrm{BEC}}-F_{\text {gas }}=\left[U_{\mathrm{BEC}}-S_{\mathrm{BEC}} T_{\mathrm{BEC}}\right]-\left[U_{\text {gas }}-S_{\text {gas }} T_{\mathrm{BEC}}\right]$ it follows that $U_{\mathrm{BEC}}<-S_{\mathrm{gas}} T_{\mathrm{BEC}}<0$, i.e. the internal energy of the condensate $U_{\mathrm{BEC}}$ is negative and, because $U_{\mathrm{BEC}}<0=U_{\text {gas }}$, gives the lowest energy value of the Bose gas. Conversely, the evaporation energy $\Delta U(M)=U_{\text {gas }}-U_{\mathrm{BEC}}>0$ has to be provided to bring $M$ from the condensed to the gaseous state. In thermodynamic terms the experimental conditions at $T_{\mathrm{BEC}}$ are as follows:

$$
\begin{gathered}
U(M)=U_{\text {gas }}=0 \quad \text { if } M \text { is gaseous and } V_{\text {gas }} / M=v_{\text {critical }}, \\
U(M)=U_{\mathrm{BEC}}<0 \quad \text { if } M \text { is a condensate and } S(M)=0, \\
\Delta U(M)=U_{\text {gas }}-U_{\mathrm{BEC}}>0 \text { if the condensate mass } M \text { evaporates. }
\end{gathered}
$$

Exact knowledge of all properties of the real Bose-Einstein condensate is a prerequisite to its experimental realisation. This can only be gained on a quantum statistical basis. The value $T_{\mathrm{BEC}}$, for example, is quantum mechanically calculated as [4]:

$$
T_{\mathrm{BEC}}=\frac{2 \pi}{[\zeta(3 / 2)]^{2 / 3}} \frac{\hbar^{2} n^{2 / 3}}{m k}
$$

with $\zeta(3 / 2)=2.612, \quad n=(M / V) / m, \quad m=$ particle mass,

$$
\hbar=h /(2 \pi) \text { Planck constant, } k \text { Boltzmann constant. }
$$

Relations (7.3) give the signs of the energy of the condensate (BEC) and of the energy expended on evaporation.

\section{Results and Discussion}

Gibbs formulated (1873) the Thermodynamic Theory on the properties of matter [1] just a few years after Andrews's pioneering observations (1869) and prior to the development of quantum physics (as of 1900). Enlisting statistical laws, the theory allows correct description of macroscopically perceived matter, a system composed of a very large number of atomic or molecular particles.

Gibbs investigated the thermodynamic equilibrium of the particle mass $M$ 
in a volume $V$. The thermal properties of the system are characterised by introducing an internal energy $U$ and entropy $S$ (Equation (4.2)), and the temperature $T$, pressure $p$ and chemical potential $\mu$ are defined by partial derivatives of $U$ and $S$. The fundamental equation $V=V(M, U, S)$ is solved by means of Equation (4.1) in the explicit form $U+V p-S T-M \mu=0$. From this follow the negative sign of the free energy,

$F=U-S T=M \mu-V p<0$ (because of $T \geq 0, \quad p \geq 0, \mu<0$ ), and the relation $U<S T$, which can be satisfied by the relations $0 \leq U<S T$ or $U<0 \leq S T$. For example, in the state chart [ $p(T, v)$-vs.- $v$ ] of a fluid, the ranges of existence of $U<0$ and $U \geq 0$ are separated from one another, on the one hand, by the critical isochor $v_{c}$ and, on the other hand, by the vapor volume line $v_{v}$ at the boundary of the two-phase region; both lines meet at the critical point. In the regions of $U \geq 0$ with, respectively, $\left[p \geq p_{c}, v \geq v_{c}\right]$ and $U>0$ with $\left[p<p_{c}, v>v_{v}\right]$ the mass exists as a gas, while in the adjacent regions of $U<0$ the mass shows more or less distinct structures. Along the line $v_{c}$ the fluid has the mean energy value $\langle U\rangle=U=0$ and shows near the critical point large fluctuations $\sqrt{\left\langle(\tilde{U})^{2}\right\rangle}$, which become detectable as macroscopic density fluctuations. Crossing the line $v_{c}$ from $U>0$ to $U<0$ occurs at a continuous change in energy $\Delta U>0$ to be removed and crossing the line $v_{v}$ requires that the energy $\Delta U=U_{v}-U=-U_{l}>0$ be extracted from the fluid. The values of the internal energy of vapor $U_{v}=M_{v} u_{v}$ and condensate $U_{l}=M_{l} u_{l}$ on the saturation lines of the two-phase region can be given in acccordance with Equations (4.6) and (4.19) as functions of the measured values of the vapor pressure $p(T)$ and densities of the condensate $v_{l}^{-1}(T)$ and vapor $v_{v}^{-1}(T)$, and can be calculated absolutely. One obtains $U_{l}<0<U_{v}$ for $T<T_{c}$ and $U_{l}=U_{v}=U=0$ for $T=T_{c}$. For $T<T_{c}$, the value of the internal energy of the two-phase fluid is always negative, $U=U_{l}+U_{v}<0$.

Because the ratio $u_{v} / u_{l}$ can be given as an andytic function of the argument $z=v_{v} / v_{l}$, its evaluation according to Equation (4.21) at the same $z$ is equal for every gas and yields the values -1 at the critical point and 0 at absolute zero. It thus follows that the energy ratios $0 \leq u_{v} /\left(u_{v}-u_{l}\right) \leq 1 / 2$,

$-1 \leq u_{l} /\left(u_{v}-u_{l}\right) \leq-1 / 2$ and $-1 \leq\left(u_{v}+u_{l}\right) /\left(u_{v}-u_{l}\right) \leq 0$ can also be universally represented as functions of $z$ for every gas.

The entropy values of the two-phase fluid can likewise be calculated absolutely in acccordance with Equations (4.3), (4.23) and (5.5) from the heat capacity data $C(M, V, T)$.

The following four observations are an indication of the thermodynamic zero of a real gas becoming evident: 1) above $\left[T_{c}, p_{c}, v_{c}\right]$ macroscopic density fluctuations appear within a very narrow temperature range (critical opalescence), 2 ) at $\left[T_{c}, p_{c}, v_{c}\right]$ the mass decomposes into a vapor component with density lower than before and a condensate component with higher density, 3) at $\left[T_{c}, p_{c}, v_{c}\right]$ any heat of evaporation vanishes, and 4) below $\left[T_{c}, p_{c}, v_{c}\right]$ an interface layer between vapor and condensate appears where on approaching 
$T \rightarrow T_{c}$ the surface tension of the condensate decreases asymptotically to 0 . The relation $U\left(T_{c}, p_{c}, v_{c}\right)=0$ is thus experimentally proved.

The fundamental Equation (4.1) is valid not only for a gas conforming to the Maxwell-Boltzmann statistics, but also for a gas subject to the Bose statistics. The statement that the internal energy of a condensate is negative therefore also applies to the internal energy of the Bose-Einstein condensate. The quantum mechanical, microscopic and thermodynamical, macroscopic formulations of the internal energy are then only valid if the two energy scales have the common absolute energy reference value 0 . The energy values above 0 are positive and those below 0 negative. A system whose particles are homogeneously distributed in the volume available has in the ground state the smallest interparticle distance $d_{\min }$, or equivalently, the number $r_{\min }<1$, the smallest entropy and the lowest energy. By virtue of this most dense particle arrangement the contribution of the binding energy to the internal energy is maximal, and since binding energy is negative energy it constitutes the lowest energy of the system. In an excited state the corresponding discrete energy level is higher. As the energy level increases stepwise, the boundary to the continuum of positive energies is reached; this boundary is assigned the energy value 0 , at which the energy contributions by attractive, binding inter-particle forces and repulsive forces are equal. The system is then in the critical state with the critical value $r=1$. The critical value $U=0$ is the common boundary value 0 of the microscopic and macroscopic energy scales. In the case of a Bose gas, the energy values at the critical transition temperature $T_{\mathrm{BEC}}$ are zero for the gas and negative for the Bose-Einstein condensate and above $T_{\mathrm{BEC}}$ they are positive. A macroscopic system has the thermodynamically determined energy zero at the critical point $\left[T_{c}, p_{c}, v_{c}\right]$. At the lowest temperature the lowest system energy is equal to the negative value of the heat of vaporization.

\section{Acknowledgements}

The author is grateful to A. M. Nicol for the English translation.

\section{References}

[1] Gibbs, J.W. (1873) A Method of Geometrical Representation of the Thermodynamic Properties of Substances by Means of Surfaces. Transactions of the Connecticut Academy, II, 382-404

[2] Stierstadt, K. (1989) Physik der Materie. VCH, Weinheim, p. 430, T15.

[3] Callen, H.B. (1960) Thermodynamics. John Wiley \& Sons, Hoboken, Preface, Chapters 2, 3, 15.3, Figures 9.4, 9.5

[4] Pethick, C.J. and Smith, H. (2004) Bose-Einstein Condensation in Dilute Gases. Cambridge University Press, Cambridge, Equation (2.23)

[5] Strunk, Ch. (2015) Moderne Thermodynamik. De Gruyter Studium, p. 32, Chapter 7.7.3 https://doi.org/10.1515/9783110371062

[6] Elsner, A. (2012) Applied Thermodynamics of the Real Gas with Respect to the Thermodynamic Zeros of the Entropy and Internal Energy. Physica B: Physics of Condensed Matter, 407, 1055-1067. https://doi.org/10.1016/j.physb.2011.12.118 
[7] Elsner, A. (2015) Thermodynamic Equilibrium of the Saturated Fluid with a Free Surface Area and the Internal Energy as a Function of the Phase-Specific Volumes and Vapor Pressure. Engineering, 7, 577-596.

https://doi.org/10.4236/eng.2015.79053

[8] Elsner, A. (2014) Thermodynamic Fit Functions of the Two-Phase Fluid and Critical Exponents. Engineering, 6, 789-826. https://doi.org/10.4236/eng.2014.612076

[9] Reif, F. (1965) Statistical Physics. Berkeley Physics Course, Volume 5, McGraw-Hill Book Company, Chapter 3.1.

Submit or recommend next manuscript to SCIRP and we will provide best service for you:

Accepting pre-submission inquiries through Email, Facebook, LinkedIn, Twitter, etc. A wide selection of journals (inclusive of 9 subjects, more than 200 journals)

Providing 24-hour high-quality service

User-friendly online submission system

Fair and swift peer-review system

Efficient typesetting and proofreading procedure

Display of the result of downloads and visits, as well as the number of cited articles Maximum dissemination of your research work

Submit your manuscript at: http://papersubmission.scirp.org/

Or contact eng@scirp.org 